Supporting Information

ES\&T Manuscript No. ES048973C

\title{
Quantification of Sequential Chlorinated Ethene Degradation Using a Reactive Transport Model Incorporating Isotope Fractionation
}

\author{
BORIS M. VAN BREUKELEN ${ }^{*}$, DANIEL HUNKELER ${ }^{\ddagger}$, AND FRANK VOLKERING ${ }^{\S}$
}

\section{Contents}

PHREEQC input file reproducing Figure 2 in article

\footnotetext{
${ }^{*}$ Corresponding author, Department of Hydrology and Geo-Environmental Sciences, Faculty of Earth and Life Sciences, Vrije Universiteit, De Boelelaan 1085, 1081 HV Amsterdam, the Netherlands, phone +31-20-5987393, fax +31-20-5989940, email: boris.van.breukelen@,falw.vu.nl

${ }^{\ddagger}$ Centre for Hydrogeology, University of Neuchâtel, Rue Emile-Argand 11, CH-2007 Neuchâtel, Switzerland

${ }^{\S}$ Tauw bv, Handelskade 11, P.O. Box 133, NL-7400 AC Deventer, The Netherlands
} 
\# ISOTOPE FRACTIONATION REACTIVE TRANSPORT MODEL (IF-RTM) ON SEQUENTIAL CHLORINATED ETHENE DEGRADATION: \# PCE (tetrachloroethene) $=>$ TCE (trichloroethene) $=>$ cis-DCE (cis-1,2-dichloroethene) $=>$ VC $($ vinylchloride $)=>$ ETH $($ ethene $)$

\# Copypaste this input file (6 pages long) in PHREEQC to reproduce Figure 2 in article.

\# PHREEQC for Windows can be downloaded free of charge at: http://www.geo.vu.nl/users/posv/phreeqc/download.html

PRINT

-reset false

SOLUTION_MASTER_SPECIES

$\begin{array}{llll}\text { Chl } & \text { Chl } & 0.0 & \text { Chl } \\ \text { Pce_1 } & \text { Pce_1 } & 0.0 & \text { Pce_1 } \\ \text { Pce_h } & \text { Pce_h } & 0.0 & \text { Pce_h } \\ \text { Tce_1 } & \text { Tce_1 } & 0.0 & \text { Tce_1 } \\ \text { Tce_h } & \text { Tce_h } & 0.0 & \text { Tce_h } \\ \text { Dce_1 } & \text { Dce_1 } & 0.0 & \text { Dce_1 } \\ \text { Dce_h } & \text { Dce_h } & 0.0 & \text { Dce_h } \\ \text { Vc_1 } & \text { Vc_1 } & 0.0 & \text { Vc_1 } \\ \text { Vc_h } & \text { Vc_h } & 0.0 & \text { Vc_h } \\ \text { Eth_1 } & \text { Eth_1 } & 0.0 & \text { Eth_1 } \\ \text { Eth_h } & \text { Eth_h } & 0.0 & \text { Eth_h }\end{array}$

\# HERE, THE SOLUTION MASTER SPECIES ARE DEFINED

\# Chloride produced during reductive dechlorination as tracer

\# 12C-PCE, "light" PCE

\# 13C-PCE, "heavy" PCE

\# 12C-TCE

\# 13C-TCE

\# 12C-DCE

\# 13C-DCE

\# 12C-VC

\# 13C-VC

\# 12C-ETH

\# 13C-ETH

SOLUTION SPECIES

Pce_1 = Pce 1

$\begin{array}{ll}\log \mathrm{k} & 0.0\end{array}$

Pce_h $=$ Pce_h

$\log \mathrm{k} \quad 0.0$

Tce_1 $=$ Tce_1 $\log \mathrm{k} \quad 0.0$

Tce $h=$ Tce $h$

$\log$ k 0.0

Dce $1=$ Dce 1

$\log \bar{k} \quad 0.0$

Dce $h=$ Dce $h$ $\log _{\mathrm{k}} 0.0$

Vc_l $=$ Vc 1 


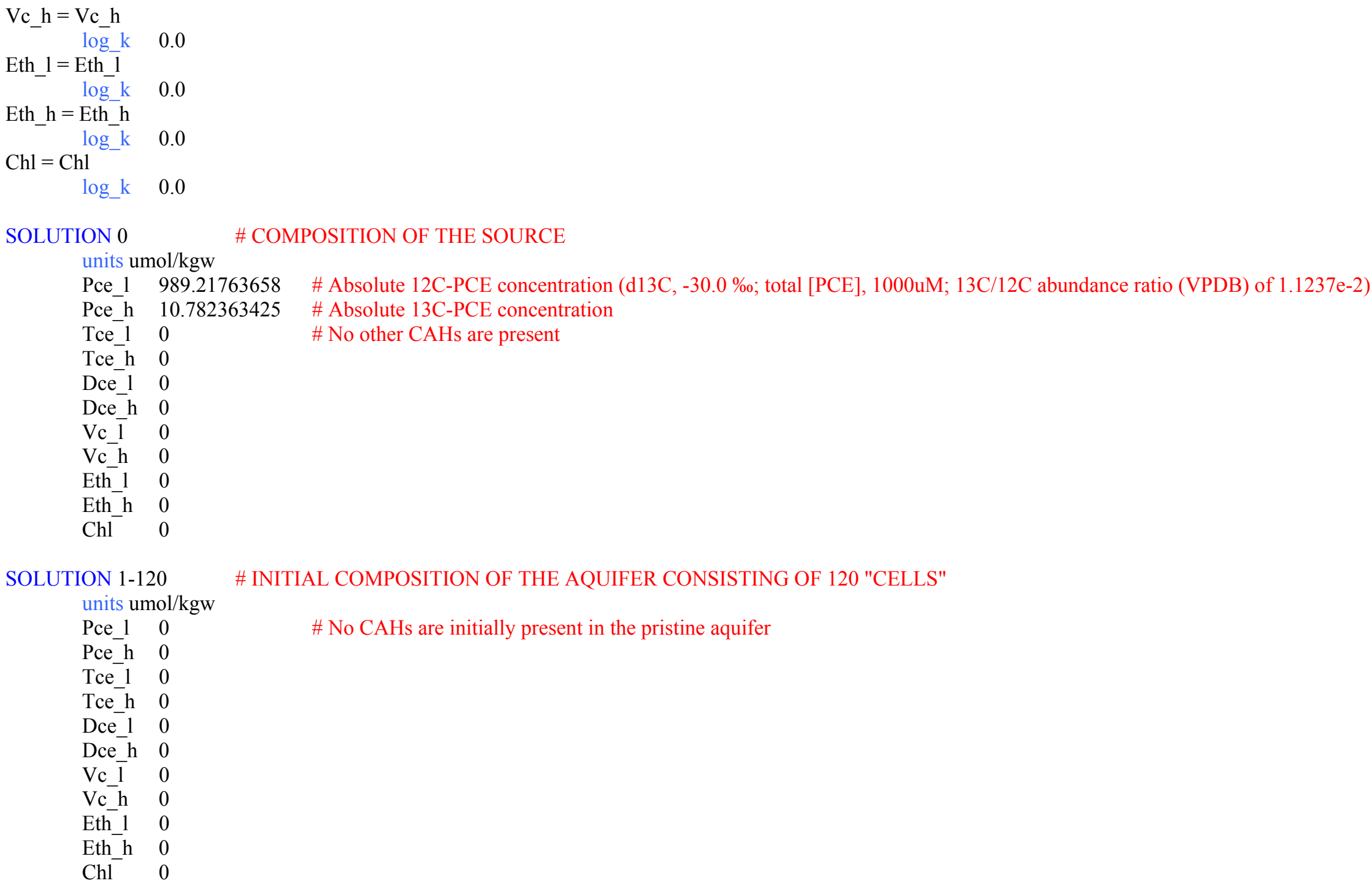




\section{KINETICS 1-120}

Pce_1_rd

Pce_h_rd -formula
-parms \# Reductive dechlorination of "light" PCE

\begin{tabular}{|c|c|}
\hline Pce_1 & Tce_1 $\quad-1$ \\
\hline $5.5 \mathrm{e}^{-}-3$ & \# first-order degradation rate per day for PCE \\
\hline
\end{tabular}

\# Reductive dechlorination of "heavy" PCE

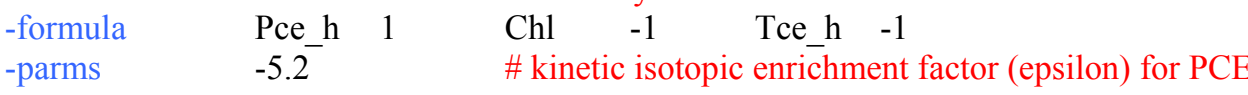

Tce_1_rd

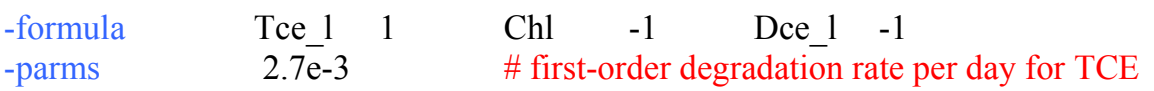

Tce_h_rd

-formula

-parms

Tce_h 1

$-8.5$

Chl $\quad-1 \quad$ Dce_h $\quad-1$

\# kinetic isotopic enrichment factor (epsilon) for TCE

Dce_1_rd

-formula

-parms

Dce_1

$1.9 \overline{\mathrm{e}}-3$

Chl $\quad-1 \quad$ Vc_l $\quad-1$

\# first-order degradation rate per day for $\mathrm{cDCE}$

Dce_h_rd

$\begin{array}{lllllll}\text {-formula } & \text { Dee_h } & 1 & \text { Chl } & -1 & \text { Vc_h } & -1\end{array}$

-parms $\quad-17.8$

\# kinetic isotopic enrichment factor (epsilon) for cDCE

Vc_1_rd

-formula

-parms

Vc_1

1.1e-3

Chl $\quad-1 \quad$ Eth_1 -1

\# first-order degradation rate per day for $\mathrm{VC}$

Vc_h_rd

Vc_h

Chl $\quad-1 \quad$ Eth_h $\quad-1$

-parms

$-23.2$

\# kinetic isotopic enrichment factor (epsilon) for $\mathrm{VC}$ 


\section{RATES}

Pce_1_rd

-start

5 if (tot("Pce_l")+tot("Pce_h")) $<1$ e-9 then goto 60

10 rate $=\operatorname{parm}(1) *\left(\operatorname{tot}(" P c e l 1 ")+\operatorname{tot}\left(" P c e \_h "\right)\right)$

20 ratio $=$ tot("Pce_l")/(tot("Pce_l")+tot("Pce_h"))

30 moles $=$-ratio $*$ rate $*$ time

40 put(rate, 1)

50 put(ratio, 2$)$

60 save moles

Pce_h_rd -end

-start

5 if (tot("Pce 1")+tot("Pce h")) $<1$ e-9 then goto 40

10 rate $=\operatorname{get}(\overline{1})$

20 ratio $=1-\operatorname{get}(2)$

30 moles $=-((\operatorname{parm}(1) / 1000)+1) *$ ratio $*$ rate $*$ time

40 save moles

Tce_1_rd

$$
\text { -end }
$$

-start

5 if (tot("Tce_l")+tot("Tce_h")) < 1e-9 then goto 60

10 rate $=\operatorname{parm}(1) *\left(\operatorname{tot}\left(" T c e \_l "\right)+\operatorname{tot}(" T c e\right.$ h")

20 ratio $=$ tot("Tce_l")/(tot("Tce_l")+tot("Tce_h"))

30 moles $=-$ ratio $*$ rate $*$ time

$40 \operatorname{put}($ rate, 3$)$

50 put(ratio, 4$)$

60 save moles

Tce_h_rd

$$
\text { -end }
$$

-start

5 if (tot("Tce_l")+tot("Tce_h")) $<1$ e-9 then goto 40 10 rate $=\operatorname{get}(3)$

20 ratio $=1-\operatorname{get}(4)$

30 moles $=-((\operatorname{parm}(1) / 1000)+1) *$ ratio $*$ rate $*$ time

40 save moles

$$
\text { -end }
$$




\section{Dce_1_rd}

-start

5 if (tot("Dce_l")+tot("Dce_h")) < 1e-9 then goto 60

10 rate $=\operatorname{parm}(1) *(\operatorname{tot}("$ Dce $1 ")+\operatorname{tot}("$ Dce $h "))$

20 ratio = tot("Dce_l")/(tot("Dce_l")+tot("Dce_h"))

30 moles $=-$ ratio $*$ rate $*$ time

40 put(rate, 5)

50 put(ratio, 6$)$

60 save moles

Dce_h_rd

$$
\text { -end }
$$

-start

5 if (tot("Dce_l")+tot("Dce_h")) $<1$ e-9 then goto 40 10 rate $=\operatorname{get}(5)$

20 ratio $=1-\operatorname{get}(6)$

30 moles $=-(($ parm $(1) / 1000)+1) *$ ratio $*$ rate $*$ time

40 save moles -end

Vc_1_rd

-start

5 if $\left(\operatorname{tot}\left(" V c \_l\right.\right.$ l")+tot("Vc_h")) $<1$ e-9 then goto 60

10 rate $=\operatorname{parm}(1)^{*}\left(\operatorname{tot}\left(" \mathrm{Vc} \_l "\right)+\operatorname{tot}\left(" V c \_h "\right)\right)$

20 ratio $=$ tot("Vc_l")/(tot("Vc_l")+tot("Vc_h"))

30 moles $=-$ ratio $*$ rate $*$ time

40 put(rate, 7 )

50 put(ratio, 8$)$

60 save moles

Vc_h_rd

$$
\text { -start }
$$

5 if $\left(\operatorname{tot}\left(" \mathrm{Vc} \_\right.\right.$l") + tot("Vc_h") $)<1 \mathrm{e}-9$ then goto 40 10 rate $=\operatorname{get}(7)$

20 ratio $=1-\operatorname{get}(8)$

30 moles $=-(($ parm $(1) / 1000)+1) *$ ratio $*$ rate $*$ time

40 save moles

$$
\text { -end }
$$




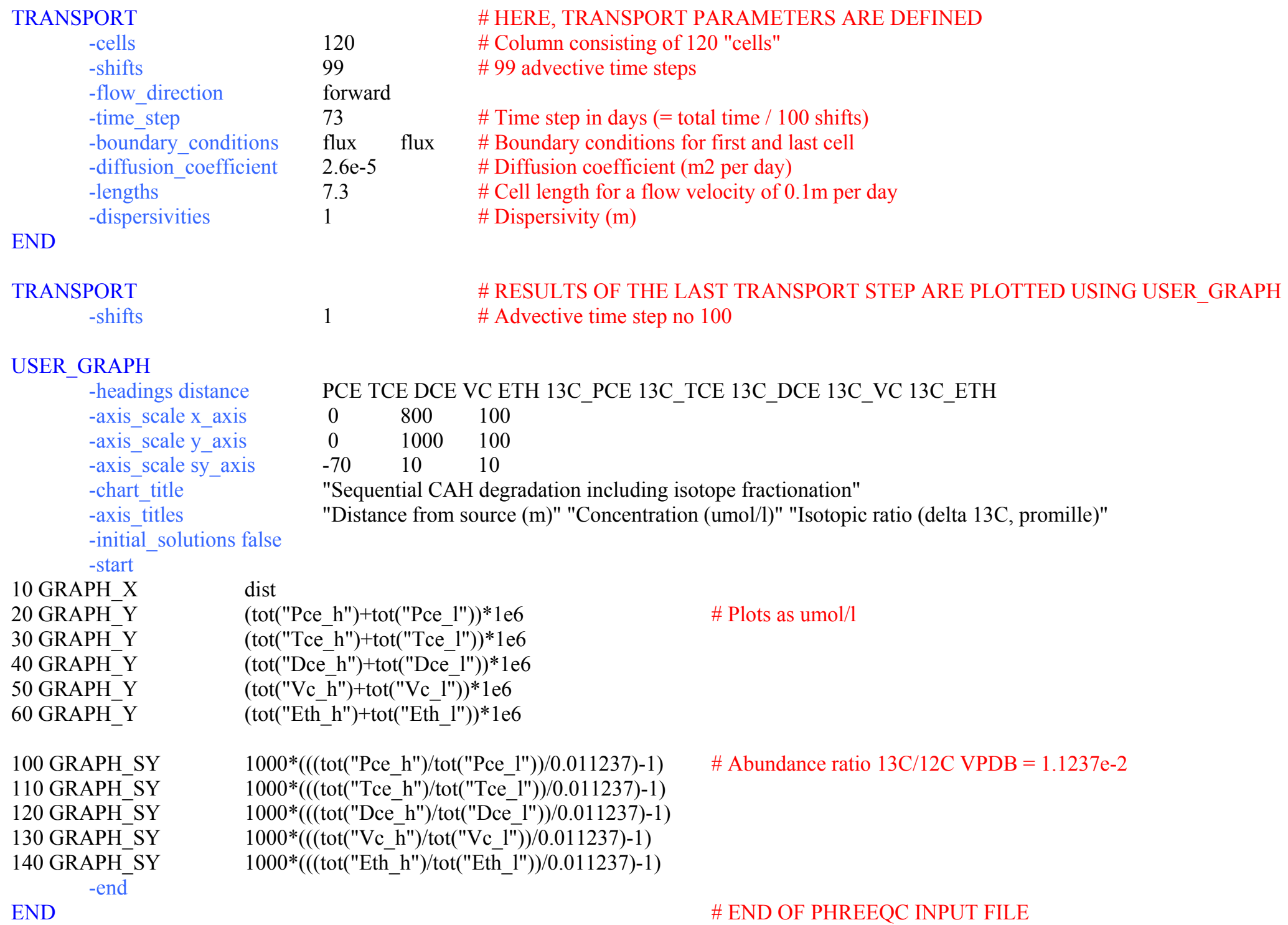

\section{USER_GRAPH}

-headings distance

-axis scale $\mathrm{x}$ axis

-axis_scale y_axis

-axis scale sy axis

-chart_title

-axis titles

-initial_solutions false

-start

\begin{abstract}
10 GRAPH X
20 GRAPH_Y

30 GRAPH Y

40 GRAPH_Y

50 GRAPH Y

60 GRAPH_Y

\section{GRAPH SY \\ 110 GRAPH_SY \\ 130 GRAPH_SY \\ 140 GRAPH_SY -end}

120 GRAPH SY

dist

(tot("Pce_h")+tot("Pce_l"))*1e6

(tot("Tce h")+tot("Tce l"))*1e6

(tot("Dce_h")+tot("Dce_l"))*1e6

(tot("Vc h")+tot("Vc l"))*1e6

(tot("Eth_h")+tot("Eth_l"))*1e6

$1000 *(($ tot("Pce_h")/tot("Pce 1") $) / 0.011237)-1)$ $1000 *(($ tot $($ "Tce_h" $) /$ tot("Tce_l" 1 - $) / 0.011237)-1)$ $1000 *((($ tot("Dce h")/tot("Dce 1"))/0.011237)-1)

$1000 *\left(\left(\left(\operatorname{tot}\left(" V c \_\bar{h} "\right) / \operatorname{tot}\left(" V c_{-} 1 "\right)\right) / 0.011237\right)-1\right)$

$1000 *(($ tot("Eth_h")/tot("Eth_l"))/0.011237)-1)

\# Plots as umol/

\# Abundance ratio 13C/12C VPDB $=1.1237 \mathrm{e}-2$

\# END OF PHREEQC INPUT FILE 